\title{
Panel Discussion I
}

\author{
Panel: F. Allard, A. Batten, E. Budding, E. Devinney, P. Eggleton, A. Hatzes, \\ I. Hubeny, W. Kley, H. Lammer, A. Linnell, V. Trimble, and R. E. Wilson
}

\section{Discussion}

M. Richards: Several talks today have expressed fuzzy boundaries to describe the objects called "stars." Is the following classification correct? Are stars restricted to objects that have masses greater than 0.089 solar masses and begin making energy with hydrogen burning? Do we include the stellar remnants: the white dwarfs and neturon stars? Do we include the brown dwarfs because they burn lithium or deuterium. We know that planets are not in this group since they have no energy production.

P. Eggleton: Well, I'll first admit I don't see this as a tremendously consuming debate and what we call them doesn't matter all that much compared to what they are. I don't think nature is necessarily going to create things that will fit in completely discrete baskets. But I do think with planets we're probably thinking of things that form in disks around stars. However, if we're thinking about brown dwarfs and things that act more like a companion around a binary star, there may not be a clear distinction. The fact that there is nuclear burning is pretty significant for a star. There might be some white dwarfs that are so evolved that they are no longer burning anything nuclear at all, they're just radiating heat, in which case they're not stars according to some people, but I would say they're still stars.

V. TRIMBLE: Planets are chemically differentiated. It makes them very hard to recognize. But that's the line for planets, chemical differentiation.

R. WiLson: Evolved stars are also chemically differentiated.

I. HuBENy: Well I think that it's merely a question of terminology since nature behaves in certain ways. For instance, from the point of view of understanding radiation from those objects it really doesn't matter whether it is a planet or brown dwarf because they are basically the same type of object. Modeling is completely the same for both those topics; the same analysis applies for everything. People like terminology, people like distinctions between objects, but I think that's a secondary problem compared to really understanding the nature of these things.

A. Batten: I think I agree with Peter. During the Prague General Assembly when we were all discussing the fate of Pluto, I suggested that astronomers could perhaps sometimes learn from philosophers, and in particular from Emmanuel Kant, who argued that we impose our categories on nature. For some purposes, we do have to define classes of objects, but really there is no essential difference between a star and a brown dwarf at one end of the range and between a brown dwarf and a planet at the other. It's our convenience that imposes these terms, and I don't think it really reflects anything real. 
W. KLEY: I would agree that it's usually not necessary to define these categories, but if I say I am working in planet formation sometimes people ask very plainly, "What is a planet?" And then you have to give some answer. It usually helps to have some categories; but I have the opinion that it is not really very useful to distinguish them by their formation mechanism since this is a priori not known. If we observe these objects now, we cannot tell whether they were formed by some gravitational instability process, whether they formed in a disk, or whether they formed somewhere else; so this is not really possible. And if we come to the initial definition of a planet where the word came from, like a wanderer in the sky, I think it's something which is not self-luminous and orbits a star that is self luminous, which is burning something. I think if we come back to this original idea of a planet, this would give a quite natural definition of it.

There is some limit, and this is something we argue about - whether we agree with the limit of 75 or 80 Jupiter masses. But there is some limit; and we would agree there is a limit between a fusor and a non-fusor. So, one could set this mass limit essentially, and one could set this as a basic limit between stars and some other objects. I am still a little confused about the limits between brown dwarfs and planets; but we can say we either have a star or we don't have a star. I think all burned out objects, previous stars, are still stars. And Pluto, well, bad luck! But Pluto is still a planet, still a dwarf planet. So it's not a minor object, it's still somewhat of a planet.

V. TRImble: I think the brown dwarfs all became disputatious, because for so long people looked hard for sub-stellar objects and didn't find any. And when finally people began to find something that was perhaps in that regime then it was briefly important to make a clean cut to say: "Yes, we have discovered sub-stellar objects." But now that there is no doubt about their existence, there is no major reason to worry about it. If you do the calculation of course as the gas cloud contracts, you get a bit of fusion and lots of things. And the question is 'Does it stop the contraction?' If it stops the contraction, then you have a star.

E. BudDing: I've come up with a couple of ideas. One is a kind of built-in argument that applies in science, which you can trace back to Newton on the basis of his analysis of half a dozen planets or planetary bodies. Newton describes his law of gravitation as being universal when speaking of a volume incredibly small compared to the now known volume of the universe. Applying that sort of concept to stars in relation to this meeting, very often we're using our ideas from the analysis of binary systems and saying, "Well, now we know what stars do."

So, let me try and put this a bit more succinctly in relation to say the primary star in the Algols. There's a line of thinking in which an Algol primary has a certain mass and a certain luminosity and therefore it can look like a main-sequence star. So we conclude that it is a main sequence star! Indeed, it looks like a main sequence star, but how can a star which has had this strong history of interactive evolution in a close binary system really be quite the same thing as a single object, which on the face of it may look quite similar. So, we should have some element of caution when we extrapolate ideas which we get from close analysis of binary systems, useful as they are, to stars as a whole. May there not be sometimes special circumstances of binarity which we might overlook?

And now the other point, one of the topics which has cropped up a couple of times today has concerned gravity darkening. The case which pushed itself forward was because of the asymmetry in the transiting minimum, and that happened to be because the axis of rotation was not in the same plane as the orbit, so there's a built-in asymmetry. The thing is that gravity darkening is always there. We also heard a lot about detailed analysis of 
limb-darkening. So, what about gravity darkening in the same degree of detailed analysis? Here we're on tricky ground, because both theoretically and observationally, it's a difficult parameter to pull out.

Getting back to the point, some years ago at a meeting in Turkey I said, "Well what about Jupiter?" There we have an object which in the far infrared is radiating on its own basis, from its own store of energy. We could check this energy distribution, this flux distribution of the surface in the far infrared and test whether a star-like body is behaving according to our expectations of gravity darkening. But, when I said how that might be done somebody said that this effort would be defeated by meteorological effects, which are more important than the effect I was trying to think about.

A. Hatzes: For me, I consider that there are 'stars' and 'the rest.' And in 'the rest' there are the brown dwarfs, the planets, etc. I always liked to show my class the nice evolutionary tracks created by Adam Burrows. You see the stars - they turn up and then they go flat; you know - hydrostatic equilibrium; and 'the rest' go down. I tell my class that 'the rest' are all alike. So, I consider these to be very similar objects.

I also worry about coming up with too much terminology, should we include remnants in the 'stars?' I don't want to get into the issue of calling them 'formerly known as stars.' If they once were stars then I think that entitles them to keep the name of star for the rest of their evolutionary status.

A. LinNELL: I think that inevitably the concept of a star needs to be just a little bit fuzzy. I think that all objects in the Kelvin-Helmholtz phase should be called stars. But hot Jupiters are not stars. So, there has to be a certain degree of fuzziness in the concept and partly it's observationally driven.

E. Devinney: I'd like to just emphasize what Al mentioned. I think that we still need to put things in context when we use each term; based on its origin or maybe the situation in which the object is found. I like the point about the philosophers (I Kant agree more), and I think that the situation reminds me about Principal Component Analysis (PCA). You have a certain coordinate system in which you define things, and then PCAs help to reduce the number of definitions you might need. I think that it's a natural evolution in terminology, you can't escape these problems, and we're not going to come up with a clear answer to that question.

R. Wilson: My idea of a star is an object that believes in the Vogt-Russell theorem at some stage in its youth. That is, it reaches an equilibrium stage early on, and at that time, what you get does not depend on how you put it together. That does not apply to planets and it doesn't apply to brown dwarfs because they do not reach an equilibrium stage.

V. TRImble: A star has an effective temperature? ...

V. TRimble: I have two micro ideas, each will fit in one sentence. One was the remark from the virtual observatory talk about how good the IUE data are. But you will remember (very few of you are as old as me) that it had to be recalibrated many many times before was that good. That is a lesson for future virtual observatories and data archives.

The other thought is: There are now an awful lot of data out there and a finite number of binary stars astronomers and there are two kinds of focuses that one can adopt: one is to look at these neat unique objects that are in fact sextuplets or something and try 
to understand them. The other is to focus on nice clean spectroscopic eclipsing detached binaries to find out as much as possible about relatively normal stars. Our database of mass-luminosity relationship, age, composition is still very very sparse. And as Dan Popper reached retirement age he worried very much that nobody was going to take over from him. Johannes Andersen did for a while but now he's a director too.

I think there's a real need for people who are willing to work on these rather basic fundamental unexciting things. I cannot resist also mentioning Roger Griffin, whose radial velocity spectrometer has enormously increased our supply of long period SBs with good orbits. It is also an ancestor of the devices that found the first exoplanet from Geneva.

A. BAtten: I thought the three opening papers by Harmanec, Guinan, and Niarchos were on well-chosen topics by well-chosen speakers. Harmanec asked provocative but fundamental questions. I was sorry that Johannes Andersen was not here as I am sure he would have mounted a spirited defense of his claims for precision in the determination of stellar masses, radii, and luminosities - although, perhaps, the discussion would have gone on all morning!

Petr Harmanec asked some very important questions. Whether or not you agree with him, it is good to ask such questions. Guinan and Niarchos, on the other hand, regaled us with exciting visions of what is to come. Vast quantities of data are expected from new space instruments and interferometry will increase the number of binaries that can be studied completely. Guinan and Niarchos and their generation are doing things my generation could only dream of. In their turn, they are dreaming of things that, very probably, the younger people here will do. We need both their optimism and Harmanec's skepticism. A good motto for this symposium, and for all astronomers, might be: "Reach for the stars, but keep your feet on the ground."

H. LAMMER: I work mainly on 'the rest' but it is still very important to understand the stars. When I listened to all the talks about the missions and telescopes and observations, I found out that it is very important that we need bright stars if we would like to learn something from the rest, or characterizing the rest. There are a lot of very exciting results from missions like CoRoT and Kepler; but the problem in all of these is that the stars are very faint so it is hard to make some follow-up observations.

We know that for instance I am working on the aeronomical part, so I am interested in the interaction between the stellar environment with the upper atmospheres of these planets, and there are a lot of good studies published on this topic. Didier Queloz mentioned the Plato mission where you have not only a simple application of CoRoT and Kepler in finding more planets, but finding planets around bright stars closer to our Sun.

Ed Guinan mentioned that access to UV wavelengths might be gone when the Hubble is not there anymore, and the UV was mentioned again in the second talk about the World Space Observatory. So, if Plato could focus on very bright stars and have a good UV telescope, it would be good for binary star science and also exoplanet characterization. So the 'rest' needs more light.

V. TRImble: European astronomers will have to campaign for these instruments in Europe. The US has declared it is interested in origins, and if you look at citations of papers, the enormous disproportion that I remarked upon a decade ago, I can now say has gotten much worse. If you want your papers cited, you're not at this meeting. The hot stuff is early universe and formation and evolution of large scale structure, and that is where US funding will go. 
A. HAtzEs: Ed Guinan mentioned that we need the great facilities like JWST. However, funding for JWST is in danger, and we should not be complacent about this. This is important even in Europe, because this is one of the great facilities that a lot of people like those who work on 'the rest' are counting on to do some great science, but we should not just assume it will be there.

E. BuDDing : One of the things I found a bit scary about the morning session more than the afternoon was the tremendous amount of information that will come out of the new generation surveys. You mentioned evolution of the universe because I do encounter a bit of this with people at the Australian telescope looking forward to the era of the Square Kilometer Array (SKA), a huge project that will create enormous amounts of information. There are regular monthly meetings going on all the time about planning what will happen with all the data, what will be used, and what science it will address.

So, we've got a lot of data coming, with many possible applications. Maybe it would be useful to think about how to get this into better focus.

A. BAtTen: I'm glad you mentioned that because when I hear predictions of 1,700,000 new spectroscopic binaries, I am relieved that I am no longer compiling catalogues of these objects!

E. Devinney: I'd like to ask the panel this question: I've been thinking about what's been happening since CoRoT and Kepler have been flying and it seems to me that this has given a tremendous boost to our field. Now we can make arguments about the need for more time on bigger telescopes for the spectroscopy that's needed. I wonder what it would have been like if Kepler and CoRoT didn't fly. What would have happened to our field? I was wondering if anyone here has any comments on that.

D. Queloz: There is a plan in Europe to build the biggest telescope in the world. It's called the European Extremely Large Telescope (ELT). This plan is about to be discussed today. I just want to mention and maybe get your reaction here that there is no plan to have an Echelle spectrograph, at least in the first generation of instruments. Are you happy with that? If this audience doesn't say anything by the time ELT will be discussed, there will not be an Echelle spectrograph.

This is the situation that is evolving: everything is trapped by infrared, imaging, multiobjects, and low resolution. Is it really useful to continue having a high resolution instrument for ELT? I would certainly love to have some feedback because we're discussing this and we have to make a decision by September or October. And this is not a clear understanding in the committee of the STC whether this needs to be pushed or not. Whatever the wavelength you want to do, is this something the community would require or not?

A. Linnell: A comment on Petr Harmanec's talk about disentangled spectra and the example of a signal for a secondary star that actually was spurious. I am a strong advocate for numerical experiments. A suggestion: Could you use the derived system parameters to construct a system with and without the contribution of the putative secondary and subject both cases to disentanglement to check the sensitivity of the procedure?

V. TRIMBLE: That's how people correct for poor resolution. 
E. BuDDING: I've had also similar concerns about numbers that have been appearing. I saw several times today temperatures of stars like 13,744. A couple of years ago somebody had collected all the different experiments to determine the value of the universal gravitational constant. And most of these values are given to around five or six digits after a decimal place with the corresponding error measure. However, if you looked across the whole table between the different experiment results there is no agreement in the third figure after the decimal place.

Similarly today, we saw the Gaussian gravitational constant, GM in combination, I think it was perhaps to twelve figures. And there was the mass of the Sun given to something like six or seven digits. Does that mean that someone has got the really true value of G? Maybe you can tell me what it is.

V. TRimble: A number of people have determined $G$ to seven significant figures; unfortunately they've got different answers. Which is precisely what you said. There was one discordant one that was significant. 6.6762 (or 3) had been accepted for a long time. I think you can now have four figures, but if you want more than that, you have to go out and orbit the Sun yourself. (I meant as a free flyer.) The temperature of Arcturus went through several thousands of degrees of change, more or less monotonic, over the 1960 s and so did the mass. And I don't know where they ended up, except it's a star.

W. KLEY: Coming back to the title of the conference, it seems to be a collection of a variety of fields: Interacting binary stars to exoplanets and essential modelling tools. So, the question arises about planets in binary stars. How do they form, how do they evolve in binary stars? In the last talk we heard from Maciej Konacki that the most close-in planets in interacting binaries don't exist because of some long-term period change and red noise. I thought this was a pity because the closest topic in the combination of the themes of this conference got lost somewhat.

It would have been theoretically very nice to have these planets because they have to survive the common envelope phase of the two stars. Or a second generation of planets comes from the debris of those stars. It was theoretically very nice, very appealing. Not that I have any answers to this or whether I say 'OK, these planets must have been formed by this mechanism or that you can actually form them by this mechanism.' The formation theory is quite critical. I thought this was a very nice connecting topic and I'm a little bit sad that this has gone away, and I am looking for some another connection of the different fields.

H. LAmmeR: When I walked around the posters I think even a single star and a hot Jupiter is something like a binary system. You have Roche lobe overflow in one case of the gas of the planet so there are a lot of physical similarities. Getting back to the title, I expected the binary community has a lot of experience and models there, which I think are very useful in this way to be applied to such types of binary systems. That's not a new classification we need because this attaches the first question, what is a star, and what is a planet? From the physics, I think there are a lot of similarities between these unique close-in hot Jupiters and stars.

V. TRImble: If the center of mass of the system is inside one of them, then the other one is a planet.

P. EgGLeton: I am purely a theorist who has never observed anything in my life, except papers written by observers. This might relate to a couple of comments here, when you 
said let's not ignore the brightest stars, even though it's nice to have data on $10^{10}$ stars that are faint and $10^{4}$ stars that are really quite bright. The fourth brightest is Capella. I was trying to model it earlier this year, and I relied upon a whole string of papers about Capella. Three of the more recent ones, one published by Roger Griffin who is a well-established figure, and another I think using the TODCOR method with Torres. Each has a velocity semi-amplitude $(K)$ value for one component that differs from the other person's K values by either 9 sigma or 14 sigma, depending on which sigma you are using.

Now, how is that possible? The short answer is that the hotter component of Capella is a rather difficult object with rather broad lines broadened by rotation that makes it rather difficult to determine the parameters. Nevertheless, whatever technique is being used to disentangle the spectra, one would hope that the standard deviation would represent something not much beyond one or two standard deviations. So there is something very fundamentally not right about some methods of getting radial velocity amplitudes.

A. BAtten: I have not read the papers to which you refer, and I am surprised that the differences between the $\mathrm{K}$ values should be so great in modern values. It is, however, as you say, extremely difficult to separate that component out from the composite spectrum for Capella.

V. TRimble: Let me quote the great physicist Fred Reines who said "Half of all three sigma results are wrong." Do you know anybody who ever overestimated his systematic errors?

R. WILSON: One important point is that I appreciate that we have boards sitting around and deciding what we can afford in putting these very expensive machines into orbit and also ground based surveys. But there is one area that I think we should make a point to afford. Recently, I went to look for the response curve of one of these survey missions and all I could find was a graphical result; and in only one publication no one else had written anything on it. Well, then I went to look for another one and there wasn't even a graphical result; just a comment that "this photometric system is believed to be sort of intermediate between such and such and another Johnson band" - not even a graph. So we wind up with these phenomenally precise observations, and we don't even know what we have an observation of! A reasonably good job has been done with the Johnson bands, Cousins bands, and Stromgren bands. However, response curves should be calculated for the filter, the optics, and what have you the whole throughput thing. It's been done for these various traditional bands, and I think we have to make it our business to have that also done for these new expensive missions.

F. Allard: I have a comment on these poor brown dwarfs that are not stars and are not planets. Some new improvement has been made in the modeling of atmospheres for $\mathrm{M}$ dwarfs and brown dwarfs. The results are in my paper.

A. Batten: We have talked about the problem regarding the accumulation of vast amounts of data. I would like to tell a little story to warn us about relying on vast amounts of digital data. Soon after William, Duke of Normandy, successfully invaded England in 1066 and seized the English Crown, he commissioned a great survey of his new kingdom for taxation purposes. The result, written in the late eleventh century, 
is known as the "Domesday Book." Some time ago, in celebration of the Book's 900th anniversary, a digital version was created. Two decades later, hardware and software had changed so much that no one could read the digital version. The original Book is still extant and can be consulted by any researcher with a serious interest in its contents!

M. RichARDS: I want to thank the panel for the interesting discussion this afternoon. 